\title{
Simulation of Wireless Sensor Networks Based on Directed Diffusion Algorithm
}

\author{
https://doi.org/10.3991/ijoe.v13i10.7747 \\ Yu Tong \\ Hubei Normal University, Hubei, China \\ 2383479257 @qq. com
}

\begin{abstract}
In order to reduce the energy consumption and data congestion caused by flooding at the initial stage of the network, the cluster head and the boundary node are used as the key nodes to reduce the data flow in the network, so that the network has better delay performance. Various clustering algorithms in wireless sensor networks and the improved method of clustering protocols are introduced. On the basis of directed diffusion algorithm, a directed diffusion protocol based on CONID clustering is studied. Through the OMNeT++ basic platform, the wireless sensor network simulation platform is built on the basis of the MiXiM module. The platform uses a hierarchical structure, and its network layer implements a directed diffusion protocol based on CONID clustering. The application layer implements the two step WLS localization algorithm. The MAC layer uses the FIexiTP protocol. The physical layer uses the simplest flat path fading model. Because the sensor has both perception and data transmission characteristics, the dual channel communication model is adopted. The platform is suitable for wireless sensor network detection, localization and tracking algorithm simulation. Through the hierarchical function of the platform, it can analyze the practicability and effectiveness of the algorithm in more detail, and has certain value. It can also be used in the simulation of cross layer protocols, and can combine several parameters to perform joint analysis of system performance. Simulation results show that the CONID based directional diffusion protocol has better delay performance than the conventional directed diffusion protocol. Finally, the performance of the two step WLS location protocol is analyzed from several perspectives, which provides an example for the platform.
\end{abstract}

Keywords-CONID clustering, directed diffusion (DD), routing algorithm, $\mathrm{OMNeT++}$ MiXiM

\section{Introduction}

Wireless sensor networks are becoming more and more widely used. It has changed the way of data collection in the past. The way that people deal with things in everyday life is developing toward intelligence, such as intelligent industry, intelligent environmental protection, intelligent disaster prevention and control, urban intelligent transportation, intelligent logistics, intelligent medical care and so on. Wireless 
sensor network (WSN) is a task-oriented wireless ad hoc network composed of several nodes. It integrates sensor technology, embedded computing technology, modern network and wireless communication technology and other technical fields. The target information is monitored in real time through various micro sensors. The information is processed by the embedded computing element and transmitted to the remote user through the wireless communication network. Wireless sensor networks (WSN) have a wide range of applications with the use of a new technology for information acquisition and processing [1]. At present, wireless sensor networks have been widely used in military, disaster warning, environmental, medical, transportation, power grid and other fields. In the field of emergency rescue, wireless sensor networks have advantages beyond other networks [2].

\section{$2 \quad$ Literature review}

As early as the beginning of the last century, With the integration of sensor components and the continuous miniaturization of electronic devices, it is possible to produce extremely inexpensive sensors [3]. These devices can monitor a variety of surrounding environments, such as temperature, pressure, humidity, soil structure, vehicle movement, noise levels, light conditions, the existence of certain targets. It has great processing power, including memory and wireless communication capabilities [4]. On the basis of reading the relevant data of routing algorithms in wireless sensor networks, the directional diffusion protocol based on CONID clustering is studied. OMNeT++ network simulation tools are used to build the wireless sensor network simulation platform. The platform refers to the TCP/IP mixed protocol stack model, which consists of application layer, network layer, MAC layer and physical layer. The application layer mainly tests the two-step weighted least squares localization algorithm. The network layer implements a data centric Directed Diffusion Protocol Based on CONID cluster. The MAC layer is tested by using FlexiTP protocol and LMAC protocol respectively, and the influence of different MAC protocols on the performance of the whole system is analyzed. Taking the two step WLS positioning algorithm as an example, the use of the platform is analyzed. Finally, simulation experiments show that the protocol can effectively reduce the data transmission delay in the network.

\section{Methods}

\subsection{Directed Diffusion Protocol}

The directional diffusion protocol is a data-centric routing protocol. The concept of gradient is introduced into the protocol to select paths that are suitable for use in wireless sensor networks [5]. It can also use data fusion and compression algorithms locally to reduce the amount of information circulating in the network. 


\subsection{LEACH protocol analysis}

The LEACH protocol is used for micro sensor network structure. The protocol uses the idea of combining the energy efficient routing based on cluster, media access and data fusion based on application, while the system achieves good performance in terms of lifetime, delay and quality of application perception. It contains a new distributed clustering technology that enables a large number of nodes to self- organize. It is suitable for self -clustering and cluster head cycle selection algorithm, which can distribute energy load evenly to all nodes [6]. It also has distributed signal processing technology to save communication resources.

\subsection{Introduction to OMNeT++ and MiXiM module}

Network simulation is a technique that uses mathematical modeling and statistical analysis to simulate network behavior to obtain specific network parameters. At present, the domestic network simulation tools are not mature enough. The researchers mainly use some foreign mainstream simulation tools, of which the representatives are NS-2, OPNET, GIoMoSim, TOSSIM, OMNeT++ and so on. The structure of $\mathrm{OMNeT}++$ is shown in figure 1 .

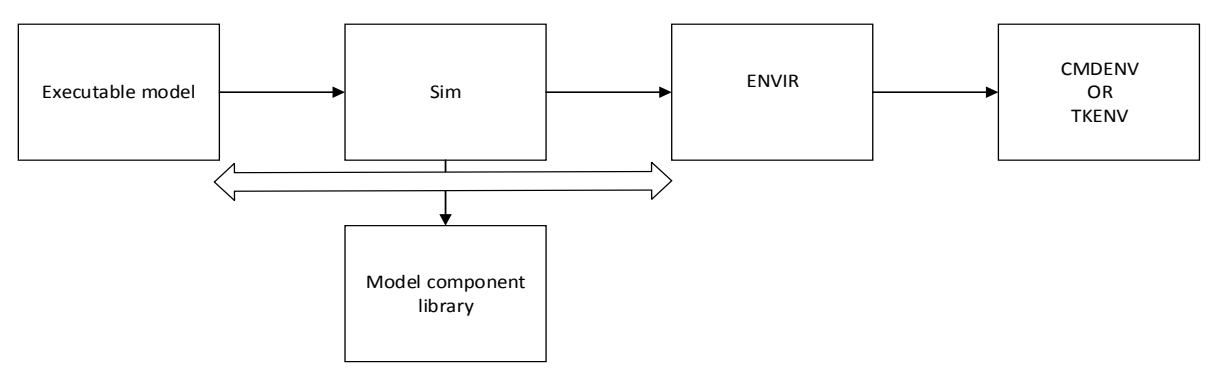

Fig. 1. OMNeT ++ internal structure

MiXiM is mainly used for the simulation of mobile and fixed wireless networks, such as wireless sensor networks, personal area networks, ad-hoc networks, vehicular networks. MiXiM focuses on the lower level of the protocol stack and provides detailed radio wave propagation and interference estimation. MiXiM model such as radio transceiver energy consumption and wireless MAC protocol refers to the TCPyP architecture [7]. Using a hierarchical architecture, many layers of modules and protocols are already available in each layer. The physical layer has a radio transceiver module, a decision maker, a channel transceiver mechanism, and a signal module. Flat fading models and logarithmic fading models have been implemented. MAC layer has BMAC, CSMA, 802.11, LMAC and other MAC protocols. The network layer has many routing protocols, such as flooding routing, adaptive probability routing and opportunistic broadcast routing. The transport layer has a convergence mechanism to achieve data aggregation function. The application layer has a traffic gener- 
ating mechanism, which can generate data streams with compound uniform distribution, exponential distribution and Poisson distribution according to the settings. In addition, there are some independent modules in MiXiM, such as ConnectionManager, used to manage the topology and connections of the entire network. The Mobility module is used to set the location of the node and to control the movement of the node.

\subsection{Introduction to simulation platform}

The platform we build uses a hierarchical structure, and the physical layer can realize the sensing function in wireless sensor networks. At the same time, it can simulate different channel states and decision methods, and realize the simulation of various network scenes. Among them, the simulation of the scene includes different scale, the movement of nodes, effectiveness of nodes and rest energy. Application layer can run detection, location and tracking algorithms. The network layer uses a directed diffusion protocol based on CONID clustering, and other routing protocols can be used. The MAC layer uses the improved Flexible-Schedule-Based TDMA Protocol (FlexiTP protocol), and also uses LMAC and other protocols to implement the media access control.

This platform can be used to analyze the performance of new protocols in wireless sensor networks, including network delay, energy efficiency and network lifetime. It can also be used to design wireless sensor networks of all sizes. The parameters of the network are analyzed to construct the most suitable network for the real environment. Of course, various algorithms on the platform is adopted to test the performance of the application layer algorithm.

\subsection{Protocol stack structure of target node}

TxAndRxMac multiple access protocol in MAC layer: The protocol implements the simplest MAC layer functionality. If the initialization state is TX (send state), the node remains in the TX state and broadcasts data passed by the application layer. If it is initialized to RX (receive state) state, the node can always maintain the RX state and receive messages sent from other nodes in the channel. Here, the initial state is set to TX. PhyLayer protocol in physical layer: The protocol implements the simplest physical layer functions, including channel and arbiter simulations. It will attenuate the received signal according to the channel model and calculate the propagation delay. The arbiter is used to determine whether the signal-to-noise ratio of the signal is above the threshold value, if it is, it is received correctly, otherwise it will be discarded. As shown in figure 2, the target node consists of application layer, network layer, MAC layer and physical layer. The letters below represent the name of the protocol used on each layer.

The target node is only in the target channel and cannot sense the messages sent by the sensor node. It is used to send messages at regular intervals, which are represented in the form of attribute pairs that can be identified by sensor nodes. 


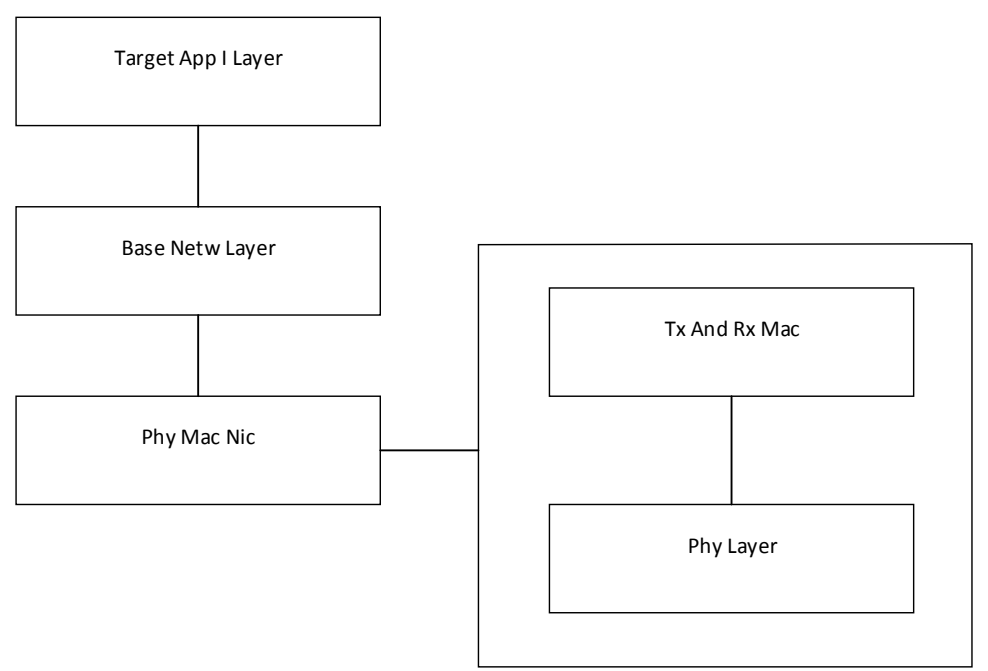

Fig. 2. Target node protocol stack

\subsection{Protocol stack structure of sensor nodes}

Because the sensor nodes not only need to apperceive the messages sent by the target nodes, but also need to apperceive the messages transmitted between the sensor nodes. Therefore, it needs to realize the function of two receiving ports. The structure at all levels is shown in figure 3.

Sensor nodes cannot only perceive the messages sent by the target nodes, but also the messages sent by sensor nodes. The perception part is different from the transmission part. The perception part is responsible for perceiving the data sent by the target node and uploading it to the application layer for caching. After a certain amount of data is cached, the data in the cache is processed (such as average) and sent to the transport application layer. The application layer of the perceptual part does not send any data down. The down connection gate is reserved for subsequent protocols only. The transmission part is responsible for the routing of the entire sensor network, and data forwarding, including clustering, sub slot and other processes. The data received from the perceptual part are processed accordingly, and the detection, location or tracking algorithms are implemented at the application level.

\subsection{Directed diffusion algorithm with fixed cluster head nodes}

Different application scenarios have different requirements for network structure. The proposed directional clustering protocol based on CONID clustering is suitable for random placement of sensor nodes, and the nodes are not controlled by the scene. For some military wireless sensor networks, the location of sensor nodes can be manually placed, and the energy of nodes can be changed periodically. For this wireless sensor network, simpler clustering and connectivity mechanisms can be used. The cluster formation algorithm is shown in figure 4. 


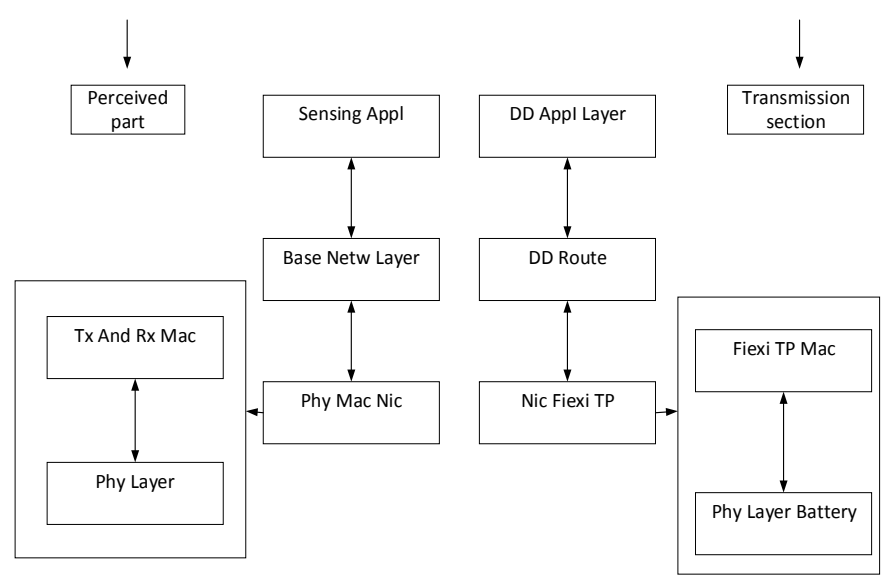

Fig. 3. Sensor node protocol stack

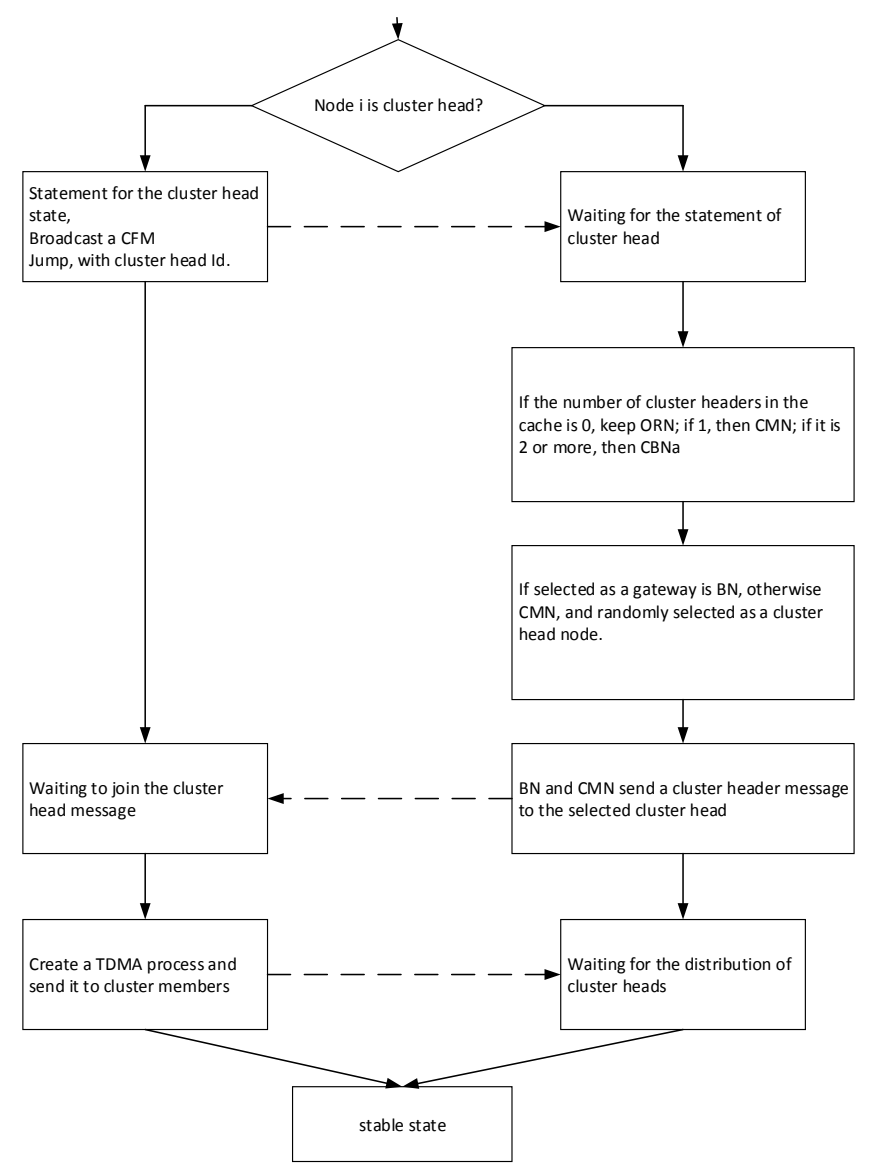

Fig. 4. Cluster formation algorithm 


\subsection{Implementation of directional diffusion algorithm based on CONID clustering}

The directed diffusion protocol based on CONID cluster is data-centric protocol, so it is necessary to generate interest messages, detect messages and data messages from application layer. The application layer of the transport section needs to send interest messages and probe messages at regular intervals. The message (the processing mechanism of timer messages) can be represented in figure 5. For messages received from the network layer, the processing mechanism of the application layer is shown in figure 6.

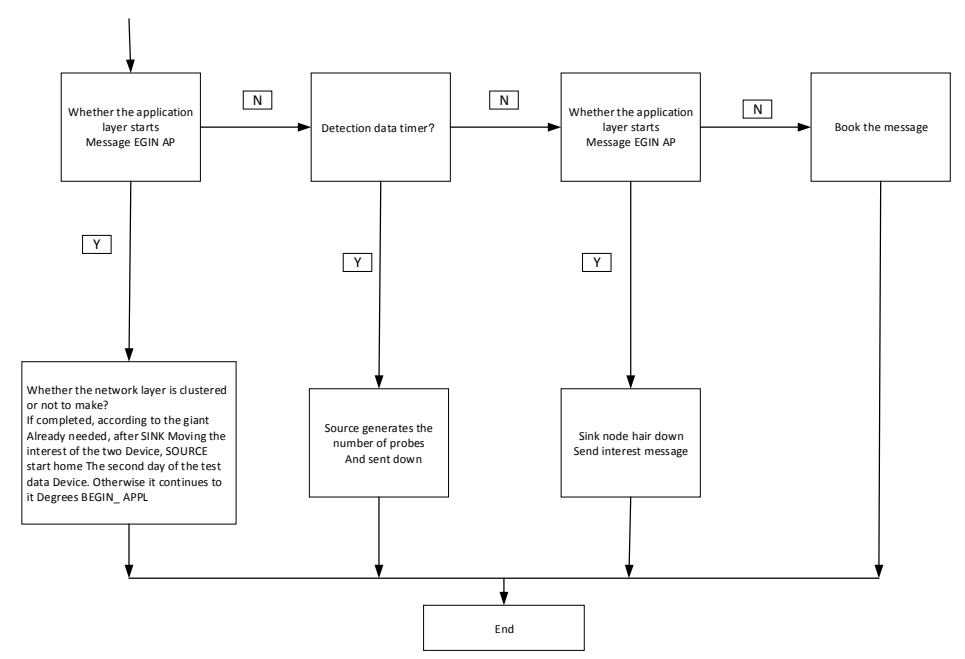

Fig. 5. Application layer from message processing

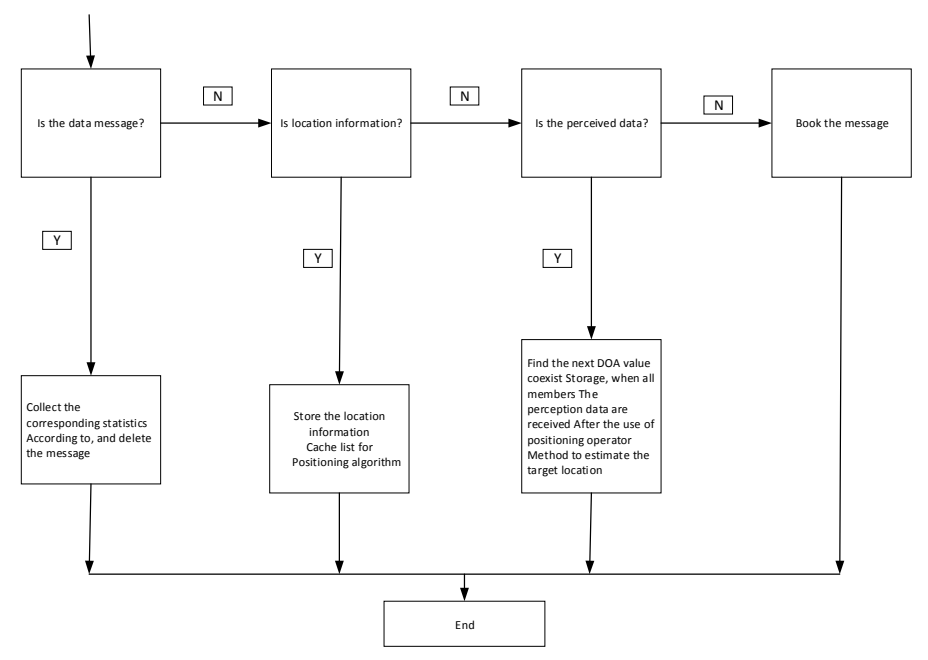

Fig. 6. Network layer upload message processing 


\section{$4 \quad$ Results}

In experiments, different number of sensor nodes are evenly distributed in the rectangular region of $160 \mathrm{~m} * 160 \mathrm{~m}$. The number of sensors is increased from 30 to 150 and an experiment is done every 30 nodes. The communication radius of each sensor is $33 \mathrm{~m}$. Figure 7 shows the influence of sensor node density on network delay. The impact of data fusion and aggregation in directed diffusion protocols and directed clustering protocols based on CONID clustering is not considered.

As shown in figure 7, whether directed diffusion protocol or directed diffusion protocol based on CONID cluster, the average delay of the network increases with the the density of sensor nodes. The delay of directional diffusion protocol increases rapidly as the density increases, while the directed diffusion protocol based on clustering rises less. With the increase of node density, the communication within the unit area of the sensor network is also increasing.

As shown in figure 8, the simulation results show that both delay will increase with the change of network size whether it is the directed diffusion protocol or the cluster oriented directed diffusion protocol.

As shown in figure 9, the average delay of the network with a distance of 20 meters is quite slow after the number of nodes in the network is greater than 90 . However, the average delay increase trend is obvious when the distance is 15 meters.

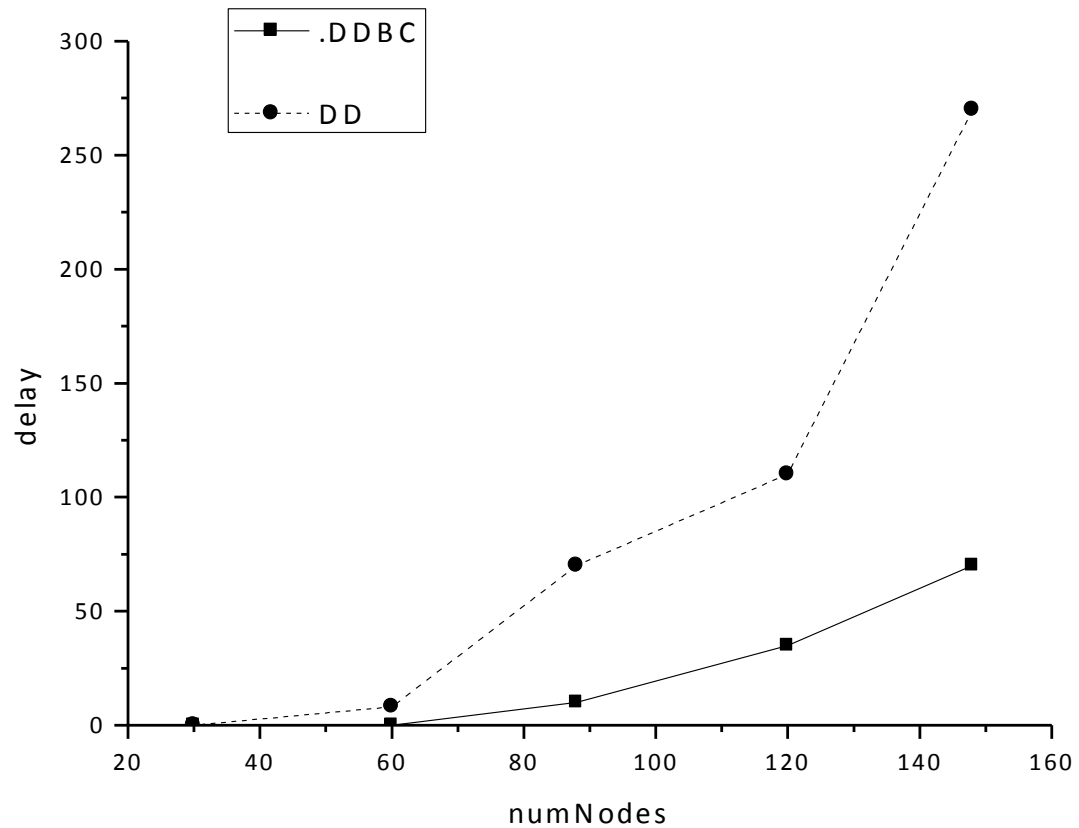

Fig. 7. Node density and delay 


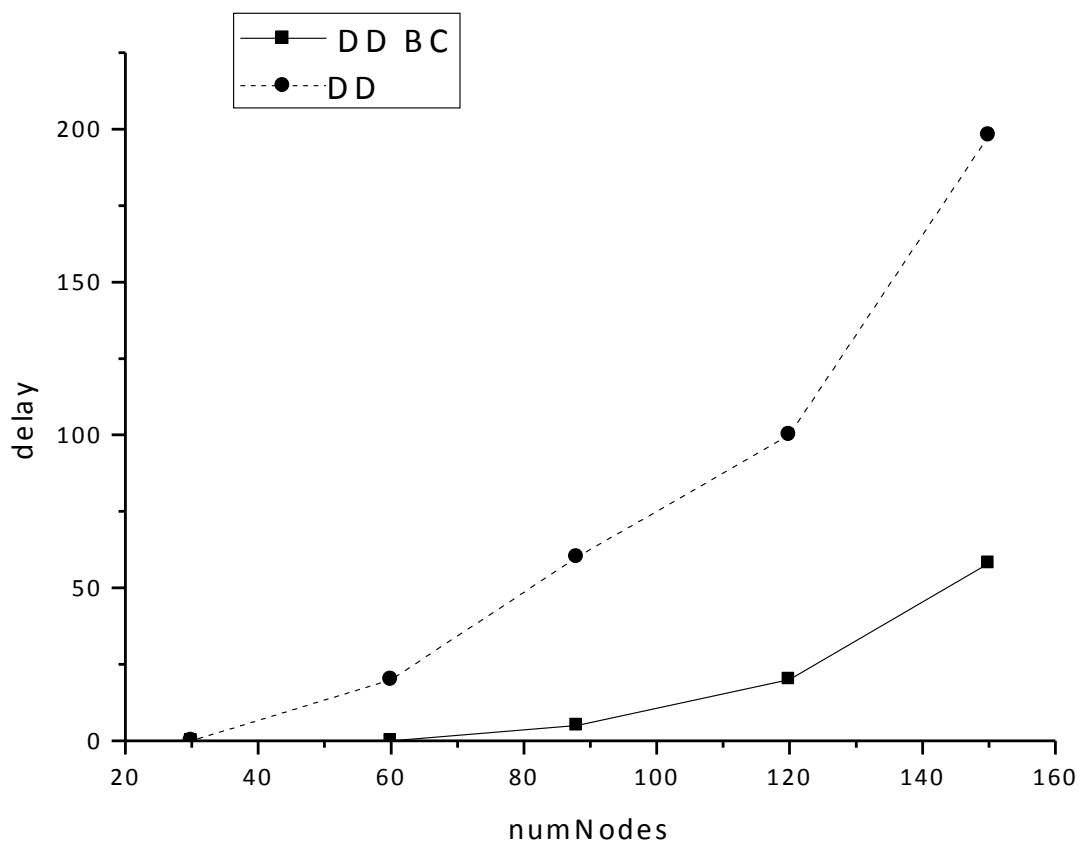

Fig. 8. Network size and delay

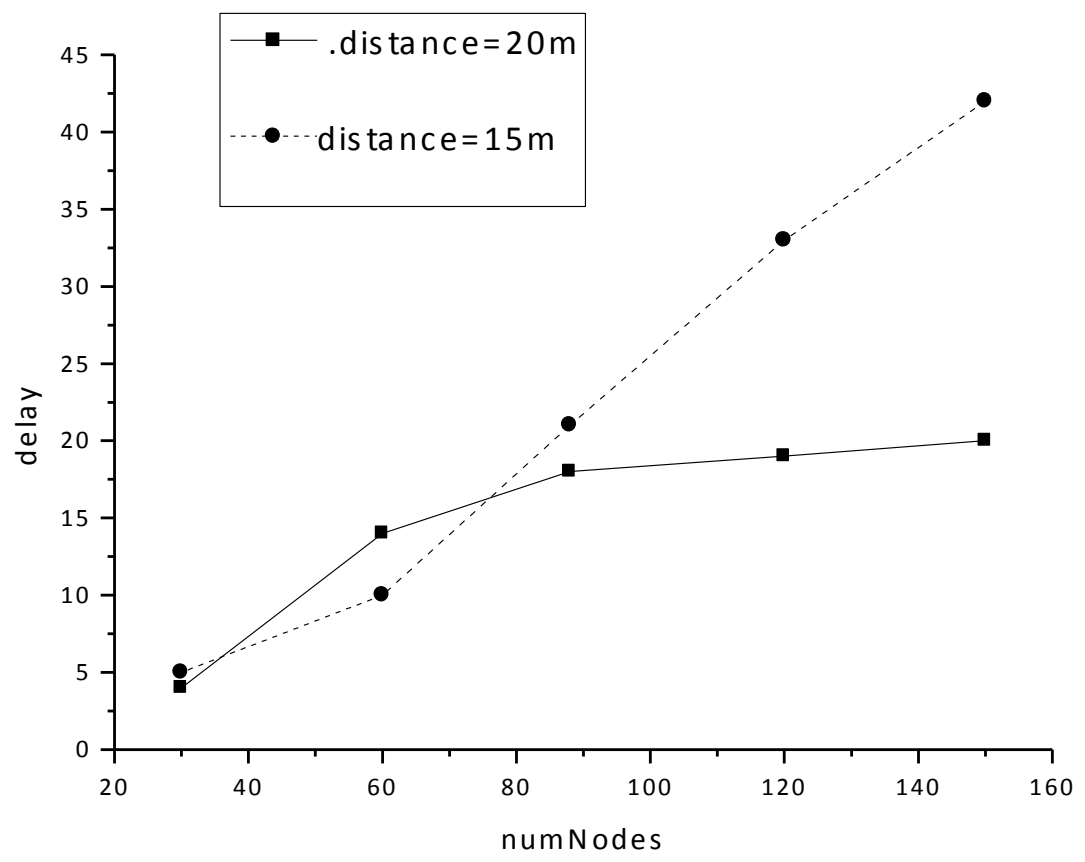

Fig. 9. Different nodes density delay graphs 
As shown in figure 10, LMAC has a much longer system delay compared to FlexiTP. FlexiTP is a time slot multiplexing multiple access protocol based on TDMA. The nodes outside the two-hop range can reuse time slots, thus reducing the length of each frame time.

As shown in figure 11, when the measured noise variance is small, the performance of the two-step WLS can almost reach the lower bound of the caramilon. This is the result when the target node is at the center of the anchor node.

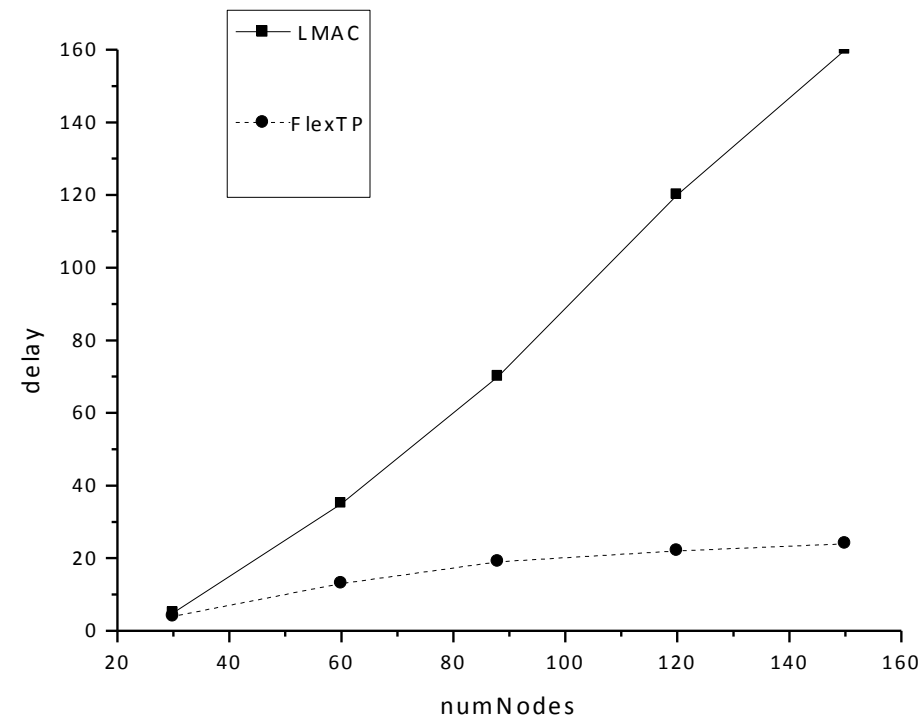

Fig. 10. Influence of Different MAC Layer Protocols on Delay

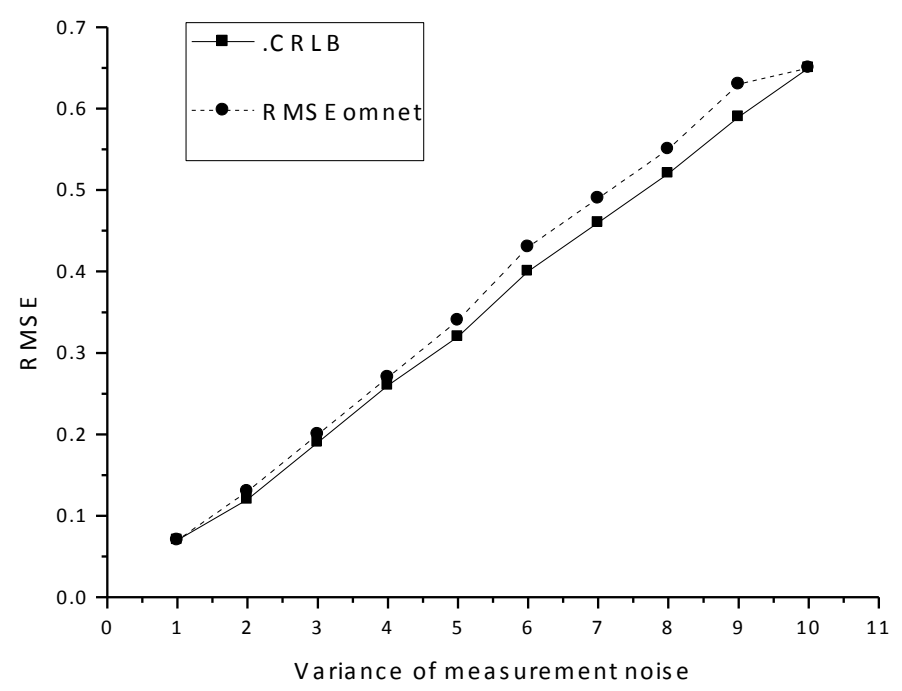

Fig. 11. Relationship between positioning accuracy and measurement error of five anchor nodes 
Figure 12 is a result obtained by using all sensor nodes for positioning. Different clusters have different angles to the target nodes, and thus the localization results are different. Sometimes serious deviations can occur, so the results cannot be used. Here is a graph showing the results of positioning each node separately. It is concluded that the simulation performance of the two step WLS positioning algorithm has a great relationship with the relative position of the target node and the anchor node, so the localization method has great limitations.

Figure 13 is an analysis diagram of node positioning accuracy of 5 anchors. Among them, the location of the target node becomes $(60,90)$, and other anchor nodes remain unchanged. As shown in the figure, the positioning performance of the algorithm is far apart from the CRLB, and the performance is not good when the target is near the anchor center.

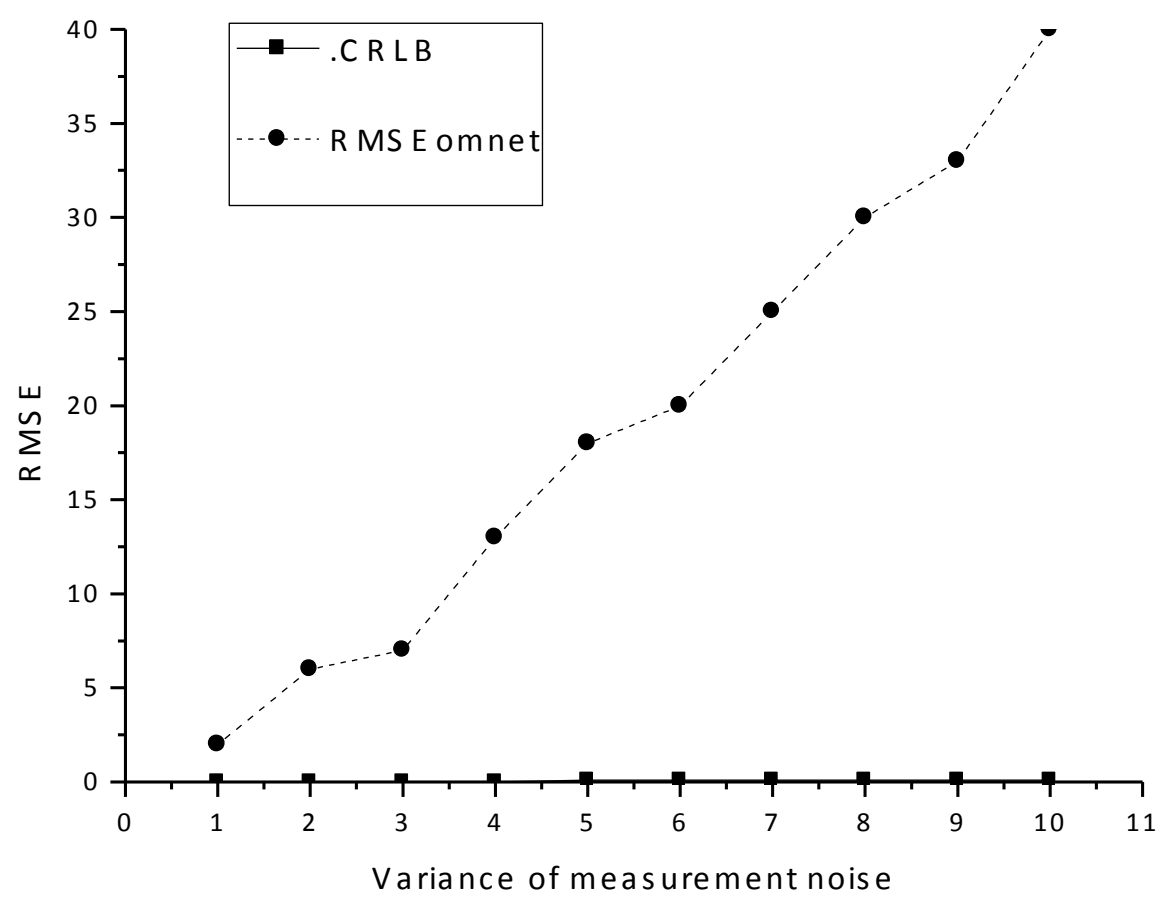

Fig. 12.The Relation between the Precision of Node and the Measurement Error 


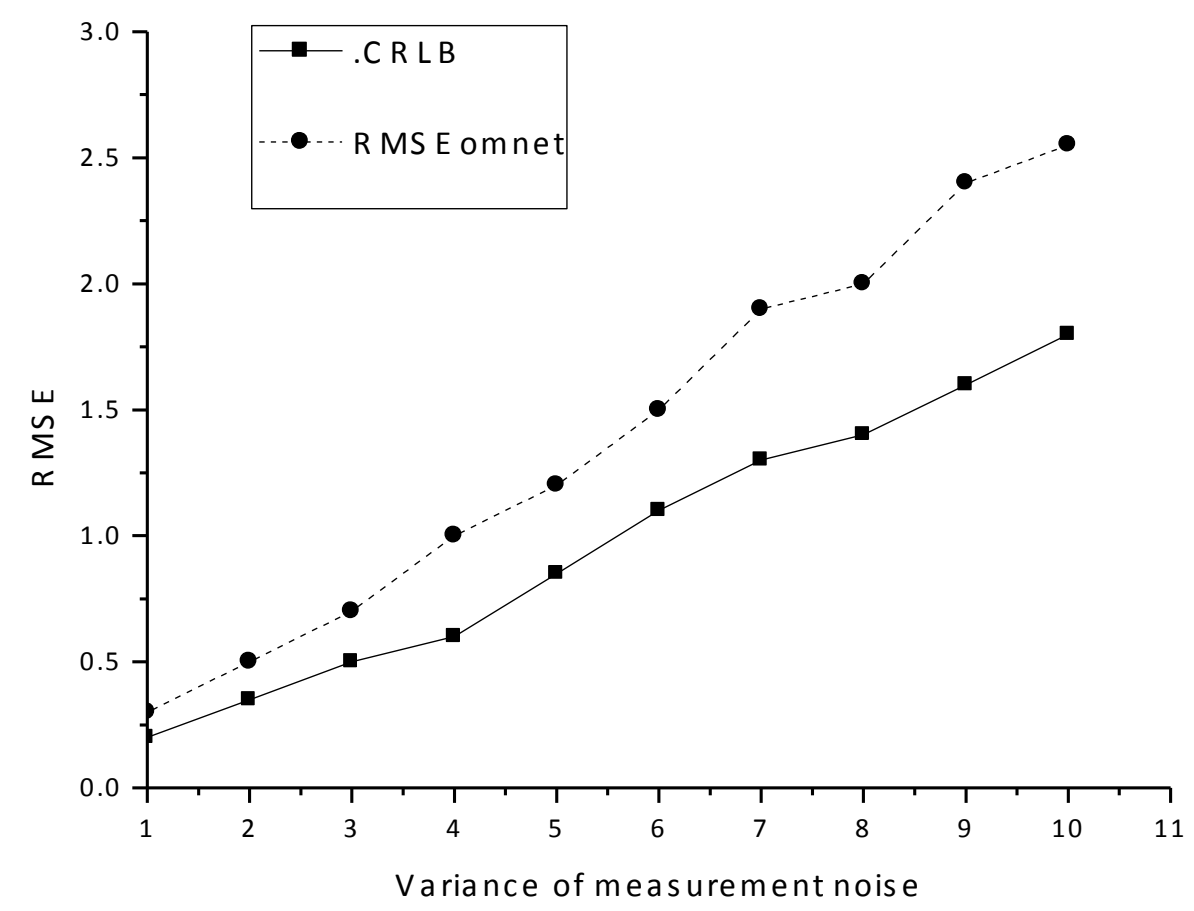

Fig. 13.The relationship between positioning accuracy and measurement error

\section{Conclusions}

Considering the needs of the simulation platform, a platform suitable for wireless sensor network simulation is created based on OMNeT++'s MiXiM platform. On this platform, the performance of different levels of algorithms is tested, including routing protocol, MAC protocol, application layer location, tracking algorithm and path fading model of physical layer. The current directed diffusion protocol based on clustering and FlexiTP protocol in MAC layer can meet the needs of wireless sensor networks, and assist the application layer simulation. Simulation results show that the directed diffusion protocol based on CONID clustering has better delay performance than directional diffusion protocol. That is to say, the algorithm not only has advantages in the speed of path establishment, but also can reduce the data transmission delay in the whole data transmission process. With the development of sensor technology and the improvement of integration capability of electronic devices, it is possible to produce cheap sensors on a large scale. Wireless sensor network has been widely used in our real life, such as smart home, fine agriculture, disaster warning and so on. It has brought great convenience to our life. While using wireless sensor networks, several issues need to be considered. How to plan a wireless sensor network? What kind of processing technology should be used and how different parameters affect wireless sensor networks? These issues have become a concern. For large-scale 
networks, it is irrational to determine the structure of the network through field experiments, which is unreasonable in terms of time, manpower, material resources and financial resources. These functions can be realized by using the related network simulation platform, including network planning, algorithm performance comparison, fault finding and so on.

\section{References}

[1] Wang J, Zhang Y, Cheng Z, et al. EMIP: energy-efficient itinerary planning for multiple mobile agents in wireless sensor network[J]. Telecommunication Systems, 2016, 62(1): 93-100. https://doi.org/10.1007/s11235-015-9985-9.

[2] Li Z, Gu Y, Tang L, et al. Fire Hazard Location Algorithm for Large Place Based on Wireless Sensor Network[J]. JCP, 2014, 9(3): 711-716. https://doi.org/10.4304/jcp.9.3.711-716.

[3] Asemani M, Esnaashari M. Learning automata based energy efficient data aggregation in wireless sensor networks[J]. Wireless Networks, 2015, 21(6): 2035-2053. https://doi.org/10.1007/s11276-015-0894-3.

[4] Zhu J, Shi C, Zhu S S, et al. Optimization of the Virtual Potential Field Based on Coverage-Enhancing Algorithm for Directional Sensor Networks[C]//Applied Mechanics and Materials. Trans Tech Publications, 2014, 651: 1882-1887. https://doi.org/10.4028/www.scientific.net/amm.651-653.1882.

[5] Mohajerani A, Gharavian D. An ant colony optimization based routing algorithm for extending network lifetime in wireless sensor networks[J]. Wireless Networks, 2016, 22(8): 2637-2647. https://doi.org/10.1007/s11276-015-1061-6.

[6] Yu C, Tu Z, Yao D, et al. Probabilistic routing algorithm based on contact duration and message redundancy in delay tolerant network[J]. International Journal of Communication Systems, 2016, 29(16): 2416-2426. https://doi.org/10.1002/dac.3030.

[7] Sergiou C, Vassiliou V, Paphitis A. Hierarchical Tree Alternative Path (HTAP) algorithm for congestion control in wireless sensor networks[J]. Ad Hoc Networks, 2013, 11(1): 257272. https://doi.org/10.1016/j.adhoc.2012.05.010.

\section{$7 \quad$ Author}

Yu Tong is with Hubei Normal University, Hubei, China (2383479257@qq.com).

Article submitted 12 September 2017. Published as resubmitted by the authors 28 October 2017. 